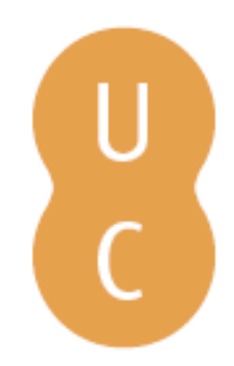

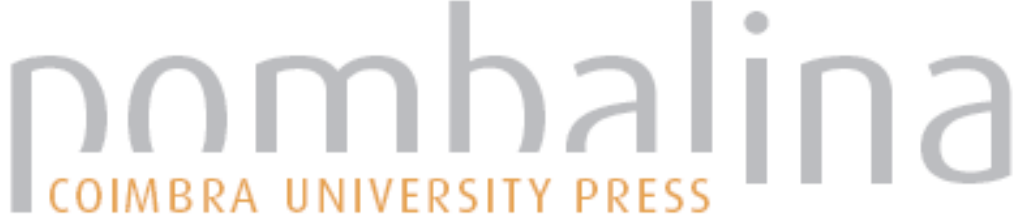

\section{The Netherlands: testimonia Belgica}

\author{
Autor(es): $\quad$ Houdt, Toon Van; Bloemendal, Jan
}

Publicado por: Imprensa da Universidade de Coimbra

URL

persistente: URI:http://hdl.handle.net/10316.2/38412

DOI: $\quad$ DOI:http://dx.doi.org/10.14195/978-989-26-0670-5_11

Accessed : $\quad$ 26-Apr-2023 16:26:06

A navegação consulta e descarregamento dos títulos inseridos nas Bibliotecas Digitais UC Digitalis, UC Pombalina e UC Impactum, pressupõem a aceitação plena e sem reservas dos Termos e Condições de Uso destas Bibliotecas Digitais, disponíveis em https://digitalis.uc.pt/pt-pt/termos.

Conforme exposto nos referidos Termos e Condições de Uso, o descarregamento de títulos de acesso restrito requer uma licença válida de autorização devendo o utilizador aceder ao(s) documento(s) a partir de um endereço de IP da instituição detentora da supramencionada licença.

Ao utilizador é apenas permitido o descarregamento para uso pessoal, pelo que o emprego do(s) título(s) descarregado(s) para outro fim, designadamente comercial, carece de autorização do respetivo autor ou editor da obra.

Na medida em que todas as obras da UC Digitalis se encontram protegidas pelo Código do Direito de Autor e Direitos Conexos e demais legislação aplicável, toda a cópia, parcial ou total, deste documento, nos casos em que é legalmente admitida, deverá conter ou fazer-se acompanhar por este aviso.

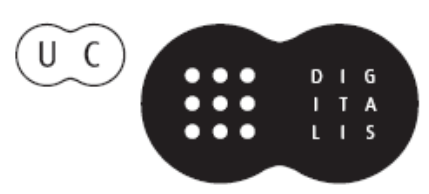


Toon Van Houdt - Latin Section, Department of Literature

(KU Leuven, Belgium)

Email: toon.vanhoudt@arts.kuleuven.be

Jan Bloemendal - Huygens Institute for the History of the Netherlands (Netherlands Academy of Arts and Sciences)

Email: jan.bloemendal@huygens.knaw.nl

\section{THE N ET H E R L A N D S}

\section{TEST I M O N I A B E L G I C A}

\section{Introduction ${ }^{1}$}

The Netherlands or the Low Countries formed a motley entity. The territory consisted of, roughly, Belgium, Luxemburg, Northern France, and the Netherlands as they exist today. In the Middle Ages, the area was divided between the dukes of Brabant and Gelre, and the counts of Holland and Flanders, while Utrecht and Liège (Luik) were ruled by the church. In the course of the 14th century, the dukes of Burgundy assembled various domains, thereby laying the foundation of a territorial unity that took shape in the 15 th century. In 1477, Mary of Burgundy married Maximilian of Austria; as a result of this marriage, the Netherlands came to be ruled by the Habsburg dynasty.

From 1512 onwards, the Burgundian domain formed one of the sixteen districts of the German Empire. In 1549, Charles V stipulated that the Burgundian district be indivisible and separated the territory from both France and the German Empire. After his death in 1556, the Low Countries were left to his son Philip II and thus became part of the Spanish branch of the Habsburg dynasty. In the meantime, the Reformation had found considerable support among the inhabitants of the Netherlands. Eager to reunite the entire country under the

\footnotetext{
${ }^{1}$ The authors wish to thank dr. Ingrid Sperber for having corrected their English.
} 
Roman-Catholic banner, Philip proposed, among other things, a drastic redistribution of dioceses. Moreover, he wanted to abolish various town privileges. All this stirred a storm of protest, which eventually led to the so-called Dutch Revolt or Eighty Years' War (1568-1648), which was conducted by the Northern troops under the initial command of William I of Orange.

During this war, the Northern Netherlands unilaterally declared themselves independent in 1581. They created the Republic of the United Netherlands, whereas the South firmly remained under the rule of the Habsburgs. During the government of the Archdukes Albert and Isabella (1598-1621), the South enjoyed some autonomy, but failed to develop into an independent nation. Meanwhile, many Protestants fled from the South to the North. Partly as a result of this migration, both countries gradually drifted apart. Whereas the Southern Netherlands became homogeneously Catholic, Protestantism increasingly prevailed in the Republic. Moreover, the 'brain drain' contributed to the economic development of the North. The role of Antwerp as a metropolis was drastically reduced, to the advantage of Amsterdam.

From the late Middle Ages until the Enlightenment, humanism was the predominant cultural movement in the Low Countries. Humanists first and foremost proclaimed a return to the sources (ad fontes): to the Bible, to texts from Greek and Roman Antiquity, and to the Church Fathers. Humanists did not aim at erudition as an end in itself; the return to the sources which they promoted and practiced had to be applied to one's own spiritual life and daily conduct. Humanism originated in Italy, and reached the Northern Netherlands through various commercial and ecclesiastical contacts. During the 15 th and 16 th centuries, Deventer, Zwolle, Kampen, and Groningen were the main humanist centres in the North. The success of (biblical) humanism was greatly furthered by another important intellectual current, the so-called Modern Devotion, which attached great importance to the inner experience of religion as the basis of one's life, the advancement of knowledge in the service of faith, and spiritual care and education. In the South, Louvain (Leuven), 
a university town since 1425 , became the most important humanist centre: from 1518 onwards, Greek, Latin and Hebrew were taught at the famous Collegium Trilingue.

Humanism engendered many outstanding representatives in the Low Countries. Here we can only mention a few of its most authoritative and influential spokesmen: Rudolphus Agricola (1443/4-1485) from Baflo near Groningen, who founded Northern Humanism; Desiderius Erasmus (1466-1536), an Augustinian secular priest and exceptionally prolific writer; Juan Luis Vives (1492-1540), a philosopher, pedagogue and social critic who left Spain for Paris and Louvain, and finally settled down in Bruges; the philologist, historiographer and philosopher Justus Lipsius who, after having taught at the university of Leiden, established in 1575, returned to the Southern Netherlands, where he became professor of Latin at the university of Louvain; and, last but not least, the lawyer, theologian and many-sided 'man of letters' Hugo Grotius (1583-1645).

Two phases can be roughly distinguished in the history of humanism in the Low Countries. As indicated above, humanism was an important cultural and even social movement during the lifetime of Agricola, Erasmus and Vives. Later the movement was institutionalized and became in a certain sense more academic, a shift which is clearly noticeable in the works of Lipsius and Grotius, as well as contemporary humanists such as the philologist and poet Daniel Heinsius (1580-1655), and the historian, theologian and philologist Gerardus Johannes Vossius (1577-1649). Humanism gradually turned into the so-called 'literae humaniores'. Leiden, where Heinsius, Vossius and Grotius had been trained by Lipsius and Josephus Justus Scaliger (1540-1609), became an internationally renowned centre of philology, which produced several important critical editions of classical authors - poets, prose-writers and philosophers alike. The language of communication was Latin, and so was Greek, albeit to a lesser extent. However, the shift from humanism to 'literae humaniores' also had a profound impact on the position of Latin, which gradually evolved from a living to an academic language. But even as a mere school and university language, Latin 
continued to play an important role. The progress made in the field of philology and its application to the Bible cleared the way for a freer approach to religion and eventually even for the Enlightenment. (Neo-)Latin letters had a lasting impact on vernacular literature, if only because many Dutch authors had enjoyed a thorough Latin education at one of the many humanist town schools that were to be found all over the Low Countries.

- $\quad$ Selective bibliography

Jozef IJsewijn, 'Humanism in the Low Countries', in Albert Rabil, Jr. (ed.), Renaissance Humanism. Foundations, Forms and Legacy, vol. 2. Humanism beyond Italy (Philadelphia, 1988), pp. 156-125; James K. Cameron, 'Humanism in the Low Countries', in Anthony Goodman - Angus MacKay (eds.), The Impact of Humanism on Western Europe (London - New York, 1990), pp. 137-163; J.P. Guépin, Het humanisme, 1350-1850 (Baarn, 1993); Jozef Janssens - Constant Mattheeussen, Renaissance in meervoud. Als dwergen op de schouders van reuzen? (Leuven, 1995), pp. 190-253; Geert Vanpaemel - Tineke Padmos (eds.), Wereldwijs. Wetenschappers rond Keizer Karel (Leuven, 2000). Ivo Schöffer e.a. (eds.), De Lage Landen van 1500 tot 1780 (Amsterdam - Brussel 1978).

\section{2. 'Nationalism' and 'Cosmopolitanism'}

\subsection{History as argument}

(1) Abraham Ortelius (1527-1598): Aurei Saeculi Imago (1596)

Having begun his career as a merchant, Ortelius decided to follow the lead of Gerardus Mercator and became a geographer and cartographer. He won lasting fame and is still remembered as the author and composer of the very first modern atlas, the Theatrum Orbis Terrarum (Antwerp, 1570).

In his Spiegel van de Gouden Tijd ('Mirror of the Golden Era'), a didactic picture book, the 'Belgian-German' (Belgo-Germanus) author Ortelius describes the customs and traditions of the ancient Germans, including (in his view) the ancient Belgians. In his work he reveals himself as an early practitioner of historical anthropology. 


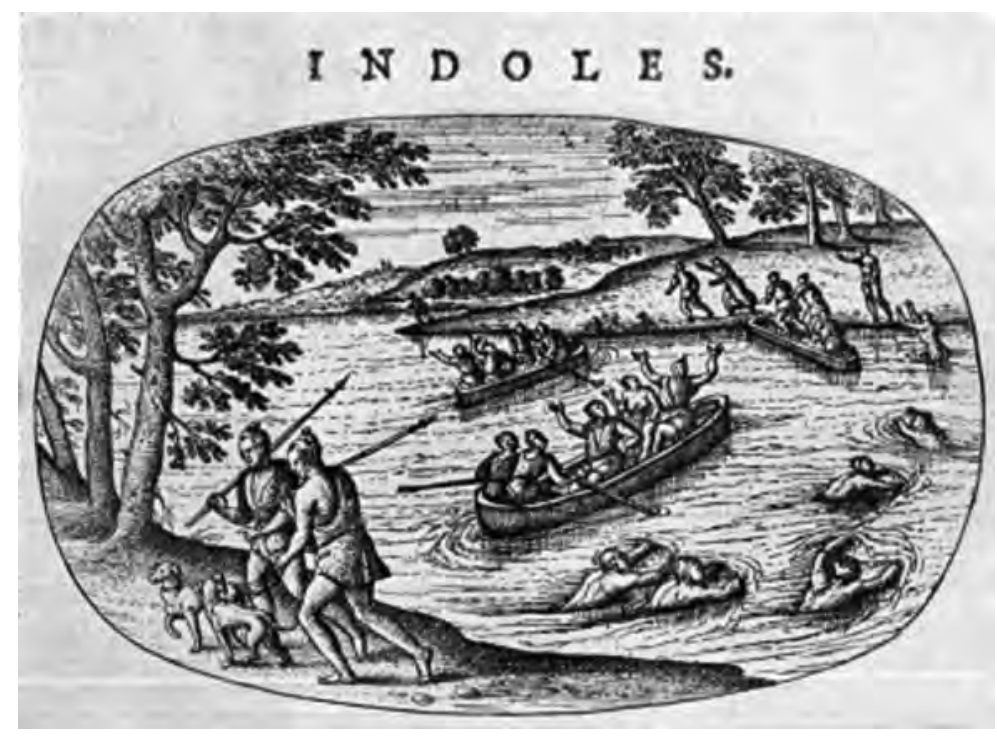

The character of the ancient Germans Engraving from Ortelius' Aurei Saeculi Imago

Sera his iuvenum venus, et intra annum vigesimum feminae notitiam habuisse in turpissimis eos habere rebus, inquit Caesar. Severa his matrimonia, singulis uxoribus contenti sunt, exceptis admodum paucis, qui non libidine, sed ob nobilitatem plurimis nuptiis ambiuntur. Dotem uxori maritus offert, boves et frenatum equum, et scutum cum framea gladioque. His matrimonii auspiciis admonetur venire se laborum periculorumque sociam, idem in pace, idem in proelio passuram ausuramque.

Paucissima adulteria, quorum poena praesens haec: accisis crinibus nudatam coram propinquis expellit domo maritus, ac per omnem vicum verbere agit, sic Tacitus. Sua quemque mater uberibus alit, neque ancillis ac nutricibus delegantur.

Numerum liberorum finire aut quemquam ex agnatis necare flagitium habetur. Nemo illic vitia ridet, nec corrumpere et corrumpi saeculum vocatur. Plusque ibi boni mores valent, quam alibi bonae leges.

- Abraham Ortelius, Aurei Saeculi Imago of Spiegel van de Gouden Tijd. Ingeleid, uitgegeven en vertaald door Joost Depuydt en Jeanine De Landtsheer (Wildert, De Carbolineum Pers, 1999). 
(2) Hugo Grotius (1583-1645): Liber de Antiquitate Reipublicae Batavicae (1610)

Grotius was, among other things, a philologist, poet and lawyer. He gained lasting fame as the founder of international and maritime law.

The young Dutch Republic was faced with a serious problem: how to legitimize its existence after having revolted against Spain, a revolt that could even be considered as a (legitimate or illegitimate) civil war? Initial attempts at creating a monarchy failed. Out of necessity, the new country established itself as a republic, with Holland as its core province. The inhabitants of this region liked to consider and present themselves as the successors of the Batavians, an ancient people governed by a council of leaders rather than by a king. As this ancient institution resembled the States, which played a major role in the political organization of the Northern Netherlands, it could easily be used to lend the Dutch Republic some sort of legitimacy:

Qua republica usi sint, Strabo satis docet, qui at Celtas omnes optimatium imperiis fuisse addictos. Celtas autem Graeci cum dicunt, tum Gallos, vicinos Batavorum, tum vero Germanos complectuntur, quorum nobilissimi guere Batavi, si quid Tacito et Plinio credimus. Et apud Gallos quidem veteres ante Romana tempore quae respublica fuerit Ambiorix ostendit, cum dicit eiusmodi esse principum imperia, ut non minus in ipsos iuris multitudo, quam ipsi in multitudinem habeant: ubi multitudinis nomine non ipsum vulgus, sed concilium cuiusque gentis primorum intelligi tum exempla apud Caesarem demonstrant, tun id quod Caesar ipse dicit, plebem nullo paene in loco esse apud Gallos, nullique adbiberi consilio, sed imperium omne intra Druides et equites consistere. Germanicas quoque gentes non sub singulorum, sed sub plurium imperio ab omni aevo vixisse ostendit illud Taciti, quo loco de antiquissimis Germanorum expeditionibus meminit: quippe regno Arsacis acrior est Germanorum libertas.

- Hugo de Groot, Liber de Antiquitate Reipublicae Batavicae, ed. E.D.E.P.O.L. (Gouda, 1995), pp. 16-19; Hugo Grotius, The Antiquity of the Batavian Republic, ed. Jan Waszink e.a., Bibliotheca Latinitatis Novae, 3 (Assen, 2000), p. 57. 


\subsection{The limits of patriotism}

(3) Joannes Ludovicus Vives (1492/1493-1540): letter of dedication of De subventione pauperum et bumanis necessitatibus (1526)

A highly original thinker, Vives was nevertheless somewhat overshadowed by the towering figure of Erasmus during his lifetime. Stemming from a family of converted Jews, Vives left his home country of Spain in 1509, in order to escape from the Inquisition. After having studied in Paris, he arrived in Bruges, where he was welcomed by the family of the Spanish merchant Bernardo Valdaura, also a converted Jew. In 1524, Vives's father was burned at the stakes in Valencia. That same year, Vives married Valdaura's daughter Margareta. His 'naturalization process' can be deemed successful: he became so attached to the Southern Netherlands that he called Flanders and Brabant his 'fatherland'. However, his foreign origin enabled him to analyze his new home country with a critical eye:

Ioannes Lodovicus Vives consulibus et senatui Brugensi salutem

Peregrini et advenae officium esse tradit Cicero in aliena re publica non esse curiosum. Ita est. Nam in res alienas curiositas ubique est odiosa; cura et amica admonitio non improbatur; quamquam neque naturae lex alienum ab homine quicquam sinit esse quod sit bominum et Christi gratia velut tenacissimum glutinum bomines omnes inter se copulavit. Et ut sit alienum aliquid, ego vero sic in civitatem hanc afficior ut in Valentiam meam nec aliter hanc nomino quam patriam; cuius iam quattuordecim annis sum incola, etsi non continenter, semper tamen buc sum solitus redire tamquam ad domum. Placuit ratio administrationis vestrae, educatio, comitas ac civilitas populi huius et incredibilis in eo quies ac iustitia per gentes celebrata. Itaque et uxorem bic duxi nec secus huic civitati consultum velim quam ei in qua quod vitae reliquum Christi benignitas fecerit traducere statuerim meque pro cive eius duco atque in cives eo sum animo quo in fratres. 
- Juan Luis Vives, De subventione pauperum sive de bumanis necessitatibus libri II. Introduction, Critical Edition, Translation and Notes by C. Matheeussen and C. Fantazzi, with the assistance of J. De Landtsheer, Selected Works of J.L. Vives (Leiden-Boston: Brill, 2002), pp. 2-3.

(4) Desiderius Erasmus (1466-1536): letter to Marcus Laurinus, 5 April 1518

An exceptionally fluent writer actively engaged in literary self-promotion, Erasmus quickly established himself as the most important representative of humanism in the Low Countries. Erasmus spent a large part of his adult life outside the Netherlands. He spent at least three years in Italy, five in England, and even more in France and Switzerland. His frequent moves led some contemporaries to denounce him as a weathercock. Erasmus's reply was simple - his real home was the Republic of Letters:

Constantiam in me desiderant homines severi, quod audierint me Basileam aditurum. Quasi vero Basileam animi causa sim profecturus, aut antehac profectus sim! Edidi Hieronymum, edidi Novum Testamentum, praeter alia multa; et ut publicis commodis inservirem, neglexi iter periculosissimum, neglexi sumptus, neglexi tantum laborum, quibus bonam valetudinis ac vitae partem attrivi. (...) Ego quoniam meapte pecunia meoque incommodo publicis inservio commodis, inconstans sum. (...) Illic, opinor, habeo sedem, ubi bibliothecam meam habeo et si quid est suppellectilis. (...) Numquam verti solum nisi aut exigente pestilentia aut valetudine cogente aut honestae rei gratia. Solam Italiam mea sponte visi, partim ut loca sacra vel semel adirem, partim ut illius regionis bibliothecis et eruditorum congressu fruerer. (...) Quod si constantiae virtus in hoc sita est, ut quam diutissime locum eundem occupes, prima laus debetur saxis ac truncis arborum, proxima conchis ac spongiis.

- P.S. Allen - H.M. Allen (eds.), Opus epistolarum Des. Erasmi Roterodami. Vol. 3 (1517-1519) (Oxonii, 1913), No. 809, pp. 266-267. R.A.B. Mynors, D.F.S. Thomson, Peter G. Bietenholz (eds.), Collected Works of Erasmus 5, Letters (Toronto, Buffalo, London, 1979), p. 363-366. Dutch translation by Dirk Sacré in Erasmus. Een portret in brieven. Vertaald en bezorgd door Jan Papy, Marc van der Poel en Dirk Sacré (Amsterdam-Mortsel, 2001), p. 32. 
(5) Hadrianus Barlandus (1486-1542): letter of dedication of Libelli tres, 4 May 1532

Barlandus was great friends with Erasmus. He taught for a while in Louvain at the Collegium Trilingue. Later he devoted himself entirely to the private education of noble youth. In his teaching programme, history lessons occupied an important place. The dedicatory letter of the Libelli tres is addressed to Arnold von Vogelsanck and Jan van Wyngaerden, canons of the chapter of Saint-Lambert in Liège (Luik). It offers an eloquent account of Barlandus's views on history, its usefulness, and the appropriate style to be applied by historiographers:

Potestne quicquam Historia dulcius esse? Per quam unam tot pulcherrimarum situs urbium, tot fluminum cursus, bortos, amoenos recessus, musarum domicilia, patrios cultus, habitus locorum, et quid quaeque ferat regio, quid quae recuset, ubi segetes, ubi veniant felicius uvae cognoscimus et domi manentes sine sumptu, sine periculo orbem ipsum terrarum nobis peragresse videmus. Eiusdem muneris utilitas nonne maxima est? Vitam, mores, facta bominum, consilia item, atque eventus rerium in medio ponit, in quae posteritas omnis tanquam in speculum inspicere possit, et vitam moresque componere. Quid bonorum animos principum ad res cum laude gerendas magis accendit inflammatque? Tyrannorum cupiditates quid magis refrenat? Dum utrique cernunt, quae in vita patrarint, futurum ut mox in orbis totius, imo saeculorum onium theatrum omnibus spectanda producantur? Nullam earum habere notitiam rerum, quae antequam nascerere, contigerunt, est semper esse puerum.

Ut praeteritis etiam saeculis vixisse videamur, sola praestat Historiarum cognitio. Absolutum Oratorem neminem dixerim, nisi et exemplis abundaverit et quamplurima noverit. In libris historicorum, ut dixi, omnis exempli documenta, velut in illustri monumento posita intuemur, unde quae imitanda sint depromere, nobisque ad imitandum proponere, quae fugienda, fugere possimus. Consulendum est itaque principibus, regibus, imperatoribus, ut historicos legant. 
- $\quad$ E. Daxhelet, Adrien Barlandus, humaniste Belge. 1486-1538 (Leuven, 1938), p. 326-328; Ari Wesseling, 'In Praise of Brabant, Holland, and the Habsburg Expansion: Barlandus' Survey of the Low Countries (1524)', in D. Sacré and G. Tournoy (eds.), Myricae. Essays on Neo-Latin Literature in Memory of J. IJsewijn, Supplementa Humanistica Lovaniensia, 16 (Leuven: University Press, 2000), pp. 229-247.

\section{(6) Desiderius Erasmus: 'Auris Batava'}

Erasmus had a rather low opinion of his countrymen, whom he considered to be uncouth and stupid. Nonetheless, the story goes that he spoke his very last words in Dutch - 'Lieve God' ('Dear God'):

\section{Auris Batava}

Quemadmodum Graeci dicunt: Bolótıov oũpov i.e. Boeoticam aurem, pro pingui crassaque, itidem Martialis Epigrammatum lib. Sexto Batavam aurem dixit agrestem, inelegantem tetricamque:

Tune es, tune, ait, ille Martialis,

Cuius nequitias iocosque novit,

Aurem qui modo non habet Batavam.

Sic enim legit Domitius Calderinus, quanquam nonnulli mutarant pro Batavam 'severam'.

Erant Batavi Germaniae populi, Cattorum pars, qui domestica seditione pulsi extrema Gallicae orae vacua cultoribus simulque insulam inter vada sitam occupavere, quam mare Oceanum a fronte, Rhenus amnis tergum ac latera circumluit; gens tum bello valida, ut quae Germanicis bellis esset exercitatissima, tum opibus pollens, quod Romana militia non exhauriretur, quandoquidem erat his cum imperio societas, ut viros tantum armaque ministrarent, quemadmodum copiosius prodidit Cornelius Tacitus lib. XX.

Convenit inter plerosque doctos, nec id refragantibus coniecturis, eam insulam, cuius meminit Tacitus, esse quam nunc Hollandiam vocant, terram mibi semper et celebrandam, et venerandam, ut cui vitae huius initium debeam. Atque utinam illi nos tam possimus honestamento vicissim esse, quam illa nobis non est poenitenda! Nam quod Martialis 
eam gentem rusticitatis insimulat, quod eandem Lucanus trucem vocat, aut nibil ad nos pertinet aut etiam laudi vertendum arbitror utrumque.

- $\quad$ Erasmus, Adagia 3535 = IV.6.35; Erasmus, Ausgewählte Werke. Vol. VII, ed. Theseria Payr (Darmstadt, 1972), p. 612-615; cf. Erasmus, Opera omnia (ASD) II.8 Adagia, ed. Felix Heinimann (Amsterdam, 1997), pp. 36-38; John N. Grant and Betty I. Knott (eds.), Collected Works of Erasmus. Vol. 36 Adages. (Toronto, 2006), pp. 235-236; Ari Wesseling, 'Are the Dutch Uncivilized? Erasmus on the Batavians and His National Identity', Erasmus of Rotterdam Society Yearbook 13 (1993), pp. 68-102.

(7) Justus Lipsius (1547-1606): De constantia (1584)

One of the main representatives of late humanism, Lipsius gained international fame thanks to his critical editions of Seneca and Tacitus, his antiquarian studies and, especially, his pivotal role in promoting Neo-Stoic philosophy.

Lipsius was born and bred in Brabant. In 1571, he decided to leave his country that was heavily afflicted by civil war. Seeking peace and tranquillity, he set out on a journey to Germany. In his famous work on constancy in times of public disaster, he evokes a long conversation which he had had with Carolus Langius, a friend from Liège, in 1572. Langius suggests a viable alternative to his journey: an innere Migration. In 1575, Lipsius returned to his beloved home village Overijse:

Ante annos aliquot cum Viennam Austriae iter haberem, fugiens patriae meae turbas, deflexi, sine Deo duce, ad Eburonum urbem, quae nec longe a via et in qua amici, quos salutare more suadebar et amore. Inter eos Carolus Langius, vir (sine fraude aut ambitione dicam) optimus doctissimusque Belgarum. Qui cum me hospitio excepisset, non omni solum comitate et benevolentia id temperavit, sed eo genere sermonum qui utiles imo salutares mibi in omne aevum essent. Ille enim fuit, ille vir, qui oculos mibi aperiret, detersa vulgarium aliquot opinionum nube, ille qui viam ostenderet, qua sine ambage pervenirem, ut cum Lucretio loquar, ad illa edita doctrina sapientium templa serena. Nam cum post meridiem fervido sole (praeceps enim iam mensis Iunius erat) domi eius in atrio inambularemus, quaesivit, ut fit, blandius de itinere meo et eius causis. Cui cum ego 
de turbis Belgarum, de insolentia praefectorum et militum, libere et vere multum dixissem, addidi postremo praetexi a me aliam, sed hanc intimam esse discessus mei causam. 'Quis enim, Langi,' inquam, 'tam firmo et tam ferreo pectore, qui diutius ferendis is malis sit? Iactamur iam tot annos, ut vides, bellorum civilium aestu, et ut in undoso mari, non uno vento agitamur turbarum seditionumque. Otium mibi cordi et quies? Tubae interpellant et strepitus armorum. Horti et rura? Miles et sicarius compellit in urbem. Itaque certum mibi, Langi, relicta hac infesta et infausta Belgica (ignoscat mibi genius patriae) terram terra commutare et quovis terrarum fugere ubi nec Pelopidarum facta neque nomen audiam.' Langius admirans et velut excitatus, 'Itane tu, Lipse,' inquit, 'abeas a nobis?' 'A vobis,' inquam, 'aut certe ab hac vita. Quod enim malorum istorum effugium, nisi in fuga? Nam ut videam ista cottidie et feram, non possum, Langi: nec chalybs aliquis mibi circa pectus.' Suspiravit ad hunc sermonem Langius et, 'Infirme adolescens,' inquit, 'quae haec mollities est? Aut quae tua mens salutem quaerentis in fuga? Turbat et aestuat patria tua, fateor, sed quae non hodie pars Europae? (...) Itaque non patria fugienda, Lipsi, sed adfectus sunt, et firmandus ita formandusque bic animus, ut quies nobis in turbis sit et pax inter media arma.'

- Justus Lipsius, De Constantia. Von der Standhaftigkeit. Lateinisch-Deutsch. Übersetzt, kommentiert und mit einem Nachwort von Florian Neuman, Excerpta classica, 16 (Mainz, Dieterich'sche Verlagsbuchhandlung, 1998), pp. 8-13; English translation: http:// www.wku.edu/ jan.garrett/lipsius1.htm (aangepast).

\section{Cultures and religions: unity and diversity}

\subsection{Tolerance and intolerance}

(8) Joannes Ludovicus Vives: letter to Franciscus Cranevelt, 1 December 1520

In November 1517, Martin Luther drew up 95 theses against the practice of indulgences and nailed them to the door of All Saints' church at Wittenberg - the start of a schism that risked splitting Europe in two 
camps. Initially, however, there was still hope for negotiation and reconciliation. But the atmosphere gradually changed, as the hardliners got the upper hand. The consequences were dire. As early as 1523, the first Lutheran heretics were burned at the stakes in Brussels. This had not yet happened in 1520, when Vives wrote the following report from Louvain, a centre of humanist studies, yet at the same time a bastion of theological orthodoxy:

\section{Vives Cranaveldio suo salutem}

Binas tuas litteras accepi diversis temporibus et datas et redditas: quae priorem habebant diem, eas posterius, specie quidem satis longas, sed revera satis breves. Nam haec est ars vestra: separare versus, ut moles fallat spectatorem non considerantem raritatem. Haec dico, quia tu mihi brevitatem obiicis, quam velim resarcias aliarum loquacitate. (...)

Theologi in tantas angustias deducunt suam rem, ac inter tot scopulos cogunt enavigare, ut difficile sit homini paulo liberiori non facere naufragium. Quae res me hominem liberum ac solutum ab ineunda via illa praeclara sane atque magnifica, quando ita volunt, sed arcta nimis et parum certe tuta absterret. Hic non per publicum modo praeconem, sed per publicum quoque carnificem medio foro, media luce, nescio quot dolia librorumne dicam Lutheri an fasciculorum - boc puto, nam liber erat unus aut alter - fasciculi permulti sunt concremati igne, de quo vereor ne magnum aliquod incendium suscitetur. Faxit Deus ut Ecclesia sua duabus rebus munita semper sit: sanis mentibus et quiete. Hanc credo affuturam, si illa non defuerit.

- J. IJsewijn e.a., 'Litterae ad Craneveldium Balduinianae. A Preliminary Edition. Part 1. Letters 1-30 (March 1520 - February 1521)', Humanistica Lovaniensia, 41 (1992), 1-85, ep. 20.

(9) Guilielmus Gnapheus: Ioannis Pistoris martyrium (1529)

In the summer of 1525, the Protestant martyr Jan de Backer or Johannes Pistorius from Woerden stood trial. In 1529, his fellow prisoner, the school rector and playwright Guilielmus Gnapheus (Willem de Volder, 1493-1568) 
gave a detailed account of the trial, which was published in Strasbourg, though not until 1546. After a lengthy biography and an overview of the interrogations, Gnapheus describes in detail how Pistorius dies a martyr's death; the description is clearly modelled on Christ's death:

Is cum iam rogum conscendisset, carnificem pronum sibi factum, et quam mortem illaturus illi erat de more deprecantem, blande excipit, et fratris nomine compellatum benigne dimittit, revocato tamen illi in memoriam vaticinio, quod superiore anno Harlemi de se dederat. Denunciarat enim fore, ex animi sui praesagio, ut in lictoris manus ob rem Evangelicam olim incideret. Caeterum, ubi iam ignibus admovebatur propius, pectus ipse suum tortori aperit, ut pulverem ille bombardicum inspergeret. Mox conscensa sella, palo tergum suum arctius appressit. Cum vero sentiret tortorem maturare iugulum, oppido quam triumphanter morti suam opprobravit victoriam: "Ubi nunc est, inquiens, inferne, gloriatio tua? ubi, mors, victoria tua? Absorpta est mors in victoria Christi." Verum enimvero, cum iugulum sibi peti a carnifice adverteret, funem quo praefocandus erat, suis ipse manibus aptabat iugulo. Hinc in coelum suspiciens: "Domine Jesu, inquit, ignosce illis, nesciunt enim quid faciunt." Et obstrangulante tortore: "O Jesu Christe, fili Dei, inquit, memento mei, miserere mei." Quo dicto, spiritus ille excludebatur.

Quam externam vim, quam praefocationis torturam nulla membrorum agitatio, nulla capitis concussio, nulla item oculorum inversio (ut fere fit) sequebatur, quod memorabile cum primis existit. Dixisses non nisi in amoenum quempiam somnum martyrem bunc nostrum solvi, ut vere solvebatur. Vivit enim cum Christo exutus malis omnibus, qui a christiani nominis confessione ne in morte quidem discessit. Quam virtutem nobis etiam largiatur in finem ille, qui est super omnia Deus benedictus in saecula. Amen.

- P. Fredericq, Corpus documentorum Inquisitionis haereticae pravitatis Neerlandicae, dl. 4. (Gent, 's Gravenhage, 1900), nr. 378, pp. 406-452 (Latin text); nr. 379, pp. 452-496 (contemporary Dutch adaptation). On Gnapheus, see Biografisch Lexicon voor de Geschiedenis van het Nederlands Protestantisme 2 (Kampen, 1978), pp. 142-144 (J. Trapman and G.J. Graafland); Biographisch-Bibliographisches Kirchenlexikon 2, cols. 256-257 (Friedrich Wilhelm Bautz). 
In the Polish city of Lublin, the Jews were said to have drained the blood from a Christian boy and then drowned him in the river. Three Jews who were suspected of having been implicated in the murder were brought before a court. At first they were acquitted, but in a second trial they were sentenced to death and executed. Jerzy Slupecki, who had studied in Holland with Gerardus Johannes Vossius and Grotius, did not know what to think of this and asked the advice of his former teacher Grotius:

Vir nobilissime,

(...) Venio ad illud de Iudaeis. Facis pro tua bonitate, quod non facile credis eorum criminationibus qui odio religionis in alios inardescunt. Pie non minus quam prudenter ab imperatoribus Honorio ac minore Theosodio constitutum est L. 14, C. de Iudaeis, ne quis cum sit innocens tanquam Iudaeus obteratur neve expositum eum ad contumelias religio qualiscunque perficiat. Quid non Christianis veteribus, quid non illis nostri prope saeculi qui a pontifice secesserant et ante Valdensibus obiecit freta potentibus praesidiis et ex accusatae partis publico odio impunitatem sperans mentiendi licentia? Iudeai ex quo patria exules infestis dominis serviunt, haud dubie ad calumnias patent. Nec tamen semper innocentes praestem, cum exsecrari Christianos fas piumque habeant, ut ex Thalmudicis aliisque libris apparet. Neque facit abstinere sunt soliti, ubi vires ipsis suas placuerunt. (...)

Ut magica aut superstitiosa taceam, cum constet puerilem sanguinem in eo se proluentibus leprae esse remedium, cuius quaesiti multi infamati sunt reges, potuere Iudaei, qui medicinam faciunt, hoc promptius eo decurrere, quo magis Christianos odere, nisi poenarum metu absterrerentur. Apparet ergo vetus esse hoc sive crimen, sive fabulem. Utrem apud nos sit, non facile dictu est. (...)

Neque miror fuisse viros graves qui Christianis omnino a quaestionibus illis violentis ac periculosis abstinendum censerent, cum certum sit in sapientissima Mosis lege fuisse incognitas et nunc Angliam sine iis 
non minus tuto quam alias gentes vivere Romaeque, dum libertas stetit, immunia ab issi fuisse civum corpora. Apud Batavos Iudaei suspecti talium facinorum non sunt, sive mitius habiti mitescunt et ipsi sive recentes advenae cautius vivunt. Certe pridem non levibus de causis Belgica omni non minus quam Gallia exacti fuere, ut de Hispania nibil dicam, ubi praeter aequum eos tractatos esse non nego. (...)

Tui amantissimus, H. Grotius

Lutetiae, XII. Decembris novi Cal. MDCXXXVI.

- F.F. Blok, Seventy-Seven Neo-Latin Letters. An Anthology (Groningen, 1985), No. 66, pp. 208-209; Grotius, Briefwisseling. Ed. B.L. Meulenbroek. Vol. VII ('s-Gravenhage, 1969), No. 2884, pp. 576-580.

\section{(11) Gerardus Joannes Vossius (1577-1649): letter to Krzystof Slupecki, 20 December 1637}

Krzystof Slupecki, who had also studied in Holland, with Gerardus Johannes Vossius and Grotius, was just as troubled as his brother Jerzy by what had happened in Lublin. He also asked his former teacher for advice. Professor Vossius gave lectures not only to his students, but also to adult citizens, and he held a consulting hour for citizens, so it was obvious that Krzystof would approach him:

(...) Indaeorum de quibus scribis miseret me prorsus. Quanto mallem sedulo ut operam darent Christiani is convertendis! Hoc fine iam Thalmudicis et Rabbinicis totum se dare coeperat oJ makarivth" Dionysius meus. Nunc plerisque omnibus terris mira eorum est paucitas qui sua tueri adversus hanc gentem sciant, pauciores etiam qui idonei videantur is expugnandis. Merito accusamus Iudaeos pertinaciae, sed nec ipsi sumus exsortes, quando apud nostros propemodum iacent Orientis litterae saepeque optimam causam lusoriis magis armis tutamur quam decretoris, quasi hoc aliud sit quam prodere veritatem. Non hoc scriberem nisi saepius observassem, quam levi amictu Iudaei exeant tela nostra, atque id hac in urbe Amstelodamensi magis etiam cognovi quam ante.(..) 
Mea nibil interest, quid in Polonia vel alibi rerum geratur. Sed horrescit animus, quoties cogito, quam parum solliciti omnes simus de salute proximi, quem malis artibus nostris magis avertimus a nobis et simul ab aeterna salute sua, saltem quantum in nobis. (...)

Amstelodami, Anno Sospitatoris nostri MDCXXVII, XIII. Kal. Ian.

Illustrissimi nominis tui Cultor,

G. J. Vossius

- F. F. Blok, Seventy-Seven Neo-Latin Letters. An Anthology (Groningen, 1985), No. 68, pp. 212-124; Boldeian Library, Ms. Rawlinson Letters 84A, ff. 96 $6_{-97}^{\mathrm{ro}}$; Gerardi Ioan. Vossii et Clarorum Virorum ad eum Epistolae (London, 1690), No. CCCLV, p. 335.

\subsection{Familiar and strange}

(12) Nicolaus Clenardus (1495-1542): letter to Jacobus Latomus, 26 March 1535

Philologist, orientalist and traveller from Diest. Upon his arrival in Portugal, Clenardus was unpleasantly surprised by the great number of (black) slaves in the country. Gradually, however, he managed to overcome his initial aversion and even started to teach them Latin as a living language. If we are to believe him, his lessons were successful:

Mancipiorum plena sunt omnia. Aethiopes et Mauri captivi omnia obeunt munia; quo genere hominum tam est referta Lusitania, ut credam Ulyssipone plures esse huiusmodi servos et servas, quam sint liberi Lusitani. Aegre reperias domum, quae non saltem ancillulam buius generis teneat. Ea foris emit quocunque opus est, lavat vestes, verrit pavimentum, fert aquam, effert suo tempore faeces domesticas et humanas, et breviter servam agit, et praeter figuram nibil differt a brutis iumentis. Nam qui ditiores sunt, plures etiam possident utriusque sexus; quidam etiam non exiguum quaestum faciunt e vernis natis, ut mibi tanquam columbas alere videantur, et adeo non offendi ancillae concubitu, ut etiam admissariis equis gaudeant, et partus ventri cedat, non vicino 
sacerdoti, aut nescio cui Aethiopi et captivo. Nam, ut hoc quoque obiter aspergam, mihi per omnem Hispaniam vere $\pi \alpha ́ v \delta \eta \mu о \varsigma$ esse Venus apparet, non minus quam olim celebrabatur apud Thebanos; maxime tamen in

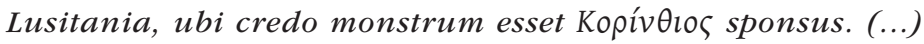

Si me ad ingenium terrae vernaculum accommodarem, ante omnia mulam alerem, et ministros quatuor. - Qui posses? - Ieiunarem domi, foris splendide triumpharem, et amarum illud sumerem pharmacum, ut plus deberem quam queam solvere. Nam ita demum perfectus absolvitur aulicus. - Et quid, quaeso, tanto opus est numero? - Etsi otium agant omnes, nemo tamen caret officio. Duo praecedunt; tertius gestat galerum; quartus pallium, si forte pluat; quintus equi capistrum; sextus boloserica sandalia; septimus scopulas illas quibus vestes repurgantur a pilis; octavus pannum quempiam, ad abstergendum equi sudorem interim, dum dominus audit sacrum aut confabulatur cum amico; nonus pectinem promit, si forte honoratior sit salutandus, ne imcomposito appareat capillitio. Quae vidimus, testamur.

His moribus tu fieri putas, ut laborem ullum ingenuo patre natus suscipiat? Etiam si dimidium proventum meorum effundam, non nanciscar ancillam liberam, aut ministrum vere ministrum. Quin et nostrates quoque, perpensa gentis consuetudine, protinus rediguntur in nobiles: pudori enim daretur, si e macello partem carnis deferrent, aut ostenderent se manibus valere. Omnia buc spectant, ut perpendas quam distent hi mores a nostratibus, ipsis quoque Lusitanis non probabiles. Verum hic gustus otii sic passim omnes corripuit, ut boni viri, velint nolint, mancipiis uti necesse habeant, et prudenter ferant quod vitare non possint.

- Correspondance de Nicolas Clénard, ed. Alphonse Roersch, tome 1 (Bruxelles, 1940), ep. 24.

(13) Augerius Busbequius (Ogier van Boesbeeck) (1520/1-1591): Legationis Turcicae epistolae quatuor (1589)

Born in Komen (Comines). After having studied at the university of Louvain, Busbequius took service with king Ferdinand I of Austria. 
Between 1554 and 1562, he was active as Ferdinand's diplomat in the empire of the Ottoman sultan Süleyman I. During his travels there, he discovered the Monumentum Ancyranum, containing the Res gestae divi Augusti.

In his Turkish letters (first comprehensive edition: 1589), Busbequius relates his adventures and experiences in the Ottoman empire. While doing so, he proves to have a keen eye for cultural differences:

Volo tibi castimoniae mulieris Turcicae exemplum commemorare. Turcae si qua alia gens uxorum pudicitiae student. Ideo conclusas servant domi atque abdunt, ut vix sol aspiciat. Quod si in publicum necessitas evocet, ita tectas, ita pannis obvolutas emittunt, ut merae larvae aut spectra occurrentibus videantur. Ipsis quidem viros per linteum aut setaceum videndi copia est, viris nulla earum corporis pars ad conspectum patet. Est enim apud eos recepta opinio non posse mulierem, quam vel minimum forma aut aetas commendet, a viro sine potiundi libidine ac proinde sine mentis labe conspici. Ob id omnes habent absconditas. Fratribus quidem earum videndi facultas permittitur. At maritorum fratribus non item. Qui sunt opulentiores aut in aliquo bonoris gradu, ea lege ducunt, ne limine unquam pedem efferant, ne quis quaeve ad eas visendi vel quacunque de causa admittatur, ne consanguineorum quidem proximi, excepto patre et matre, qui die paschatis visere filiam non probibentur. Pro quo, si mulieres eminenti dignitate patrem habeant, aut dotem attulerunt grandiorem, repromittunt vicissim mariti se nullas habituros pellices, sed eis solis contentos fore. Alioquin Turcas, quot velint ad iustas uxores pellices assumere, nulla lex vetat. Neque ullum inter harum et illarum liberos honoris discrimen, eodemque iure censentur. Pellices vero aut sibi emunt, aut bello acquirunt; quarum ubi taedet, nibil impedit, quo minus in forum productas vendant. Liberis tamen susceptis libertatem consequuntur.

- Augerius Gislenus Busbequius, Legationis Turcicae epistolae quatuor - Vier brieven over het gezantschap naar Turkije. Uitgave, inleiding en aantekeningen van Zweder von Martels, vertaling van Michel Goldsteen (Hilversum, 1994), ep. 3, pp. 186-187. 


\subsection{Towards a European pattern of civilization}

(14) Desiderius Erasmus: De civilitate morum puerilium libellus (1530)

In his brief treatise on good manners for youngsters, Erasmus advocates the need for a European code of conduct that transcends, at least to a certain extent, regional and social differences. This is not to say that the author is blind to the many particular customs and habits which he got to know during his travels through Europe. Some of those he approves of, others he strongly rejects, as he is of the opinion that they violate natural good taste or are harmful to the health. In general, he has a strong preference for universally recognizable manners that can - and should - be accepted on reasonable grounds:

In summa dictum est de corpore, nunc de cultu paucis, eo quod vestis quodammodo corporis corpus est et ex hac quoque licet habitum animi conicere. Quanquam bic certus praescribi modus non potest, eo quod non omnium par est vel fortuna vel dignitas, nec apud omnes nationes eadem decora sunt aut indecora, postremo nec omnibus seculis eadem placent displicentve. Unde quemadmodum in aliis multis, ita bic quoque nonnibil

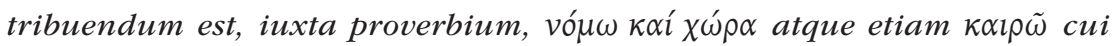
servire iubent sapientes. Est tamen in hisce varietatibus, quod per se sit bonestum aut secus, velut illa quae nullum habent usum, cui paratur vestis. Prolixas trabere caudas in foeminis ridetur, in viris improbatur, an cardinales et episcopos deceat aliis aestimandum relinquo. Mulctitia nunquam non probro data sunt tum viris, tum foeminis, quandoquidem bic est alter vestis usus, ut ea tegat quae impudice ostenduntur oculis hominum. Olim habebatur parum virile discinctum esse, nunc idem nemini vicio vertitur, quod indusiis, subuculis et caligis repertis tegantur pudenda, etiam si diffluat tunica. Alioqui vestis brevior quam ut inclinanti tegat partes quibus debetur honos, nusquam non inbonesta est. Dissecare vestem amentium est, picturatis ac versicoloribus uti morionum est ac simiorum. Ergo pro modo facultatum ac dignitatis proque regione et more adsit cultui mundicies, nec sordibus notabilis, nec luxum aut lasciviam aut fastum prae se ferens. 
- Desiderius Erasmus, Opvoeding. Vertaald en toegelicht door Jeanine De Landtsheer en Bé Breij (Amsterdam, 2006), pp. 17-18; La civilité puérile d'Erasme. Traduction, édition et introduction par Franz Bierlaire, Notulae Erasmianae, 3 (Bruxelles, 1999), pp. 46-47; J. K. Sowards (ed.), Collected Works of Erasmus, 25 (Toronto - Buffalo - London, 1985), pp. 278-279.

\section{Cultural heritage}

\subsection{Academic life}

\section{(15) Justus Lipsius: letter to Philippe de Lannoy, 3 April 1578}

Nowadays, students have recourse to Erasmus and Socrates grants to visit universities abroad. In early modern times, too, youngsters travelled to distant countries, especially to Italy, in order to study and develop themselves. In the following letter, Lipsius gives an early modern 'Erasmus student' a good piece of advice:

Philippo Lanoyo, nobilissimo iuveni, S[alutem] D[ico].

In Italiam cogitare te audio, nobilissime iuvenum, et audio volens. Placet ea mens, quam agnatam scio non nisi optimae cuique menti. Humiles istae et plebeiae animae domi resident et adfixae sunt suae terrae; illa divinior est quae caelum imitatur et gaudet motu. Itaque sive prsico sive nostro aevo peregrinati fere sunt viri magni. Sed ista hic cautio: ut id fiat non cum voluptate solum, sed cum fructu. Vagari, lustrare, discurrere quivis potest; pauci indagare, discere, id est: vere peregrinari. Ut tu id possis, finem verum fructumque ante omnia mibi porpone quem is petitum. (...)

Duo, nisi fallor: utilitatem et voluptatem. (...) Ac de voluptate aliquid dicere vacuum sit, cum ea sponte obvia nec quisquam tam lapis aut frutex quem non titillet ac flectat amoena illa variaque spectio gentium, 
urbium, locorum. Natura scilicet illuc aurigatur nec duce opus ulla ratione. At in utilitate laborandum est, quam nec secuti omnes nec qui secuti adsecuti. Eam tibi ingero et triplici hoc limite designo: prudentiae, scientiae, morum. Haec enim omnia peregrinatio adferre debet, si consilio, si rite instituta.

- Iusti Lipsi Epistolae, pars I: 1564-1583, eds. A. Gerlo - M. A. Nauwelaerts - H. D. L. Vervliet (Brussel, 1978), pp. 197-202, no 90; Justus Lipsius, Brieven aan studenten: de Romereis, honden en geleerden, drinkebroers en smulpapen. Vert. J. Papy (Leuven, 2006); N. Doiron, L'Art de voyager. Le déplacement à l'époque classique (Sainte-Foy-Paris, 1995), pp. 211-212 (Traduction par Anthoine Brun, Lyon, 1619).

\section{(16) Constantijn Huygens (1596-1687): poems}

The 'bourgeois-gentilhomme' Constantijn Huygens was active as a diplomat, poet, secretary to the princes of Orange, composer and musician.

Quite often Huygens writes with his tongue in his cheek. This is notably the case when he describes his travel to Rome:

\section{ITALIA DECOLOR}

Hesperii Manes, Mundi morientis imago

Praevia, faex rerum, splendida barbaries,

Marcide flos, cinis, umbra tui, salve Itala Tellus,

Indigenam Patriae frigidioris habes,

Frigidioris habes, non deterioris alumnum,

Hospitii sociâ qui rogo jura fide.

Illa per ingenteis olim dilapsa Triumphos

Illa super geminum Fama levata Polum,

Qua Caput atque Orbis late dominator ocellus

(10) Diceris, hos oculos impulit, hosce pedes,

Iussit ab Arctoïs discedere littoris undis,

Qua Batavi fontem nescit arena soli,

Scandere monstrosum per inhospita nubila culmen,

Ferre per aestivas torpida membra nives

(15) Iussit, et ô vanis utinam ne lapsa revolvens 
Luserit ignarum rumor imaginibus!

Infelix, at enim quam prisci rara decoris

Semina, pauca virum, nulla vigoris habes,

Nulla pudoris et ingeniti (fas dicere) bruto

Luminis ingeniti quas alis ipsa feris.

Impia divinos, specie pietatis, honores

Eripis immenso qui videt ista Deo;

Quod colis ipsa creas, quod edis colis, horreo dictis,

Mente Creatorem non capis, ore teris;

(25) Cui gemitus de corde pios, cui viscera debes,

Contritique sacras pectoris exuvias,

Dona viris, pueris spernenda crepundia profers,

Aera, faces, tabulas, marmora, thura, sonos.

Improba, cognatas agis in sua viscera dextras,

(30) Tota rubes fratrum sanguine, tota Patrum:

Flagra, sicas, aconita doces, non praelia, civem,

Ivit in acceptos fraus homicida jocos.

Luxu, foeda, fluis; quicquid congesta dederunt

Saecula vel patras una vel exsuperas;

(35) Non gladium raptor, non novit adultera Legem,

Non Sodomae sordes, non pice digna Venus.

Plura jubet calamus, jubet indignatio plura,

Ni vetet ingenuus plura referre pudor.

Sisto manum, pede linquo tuos, indigna, Penates,

(40) Forsitan bis olim qui capiatur erit,

Quo sociae veteres Romae placuere Batavi

Candor, et egregius mentis ad alta vigor

Fervet adbuc, studiisque idem Constanter abborret,

Roma, sed heu! quam nunc altera Roma tuis.

(45) En reducem dilecta tenes Hollandia Civem;

Da subeat veteres non novus hospes agros.

Rettulerint alii insanae novitatis amorem,

Italiae solus taedia sola fero.

Clavennae in Rhaetis. Iulio. 
- Constantijn Huygens, Latijnse gedichten 1607-1620. Ed. T.L. ter Meer (Den Haag, 2004), p. 202, no. 127. Constantijn Huygens, Gedichten, ed. J.A. Worp (Groningen, 1893-1899), vol 1, pp. 186-187.

\subsection{Sciences}

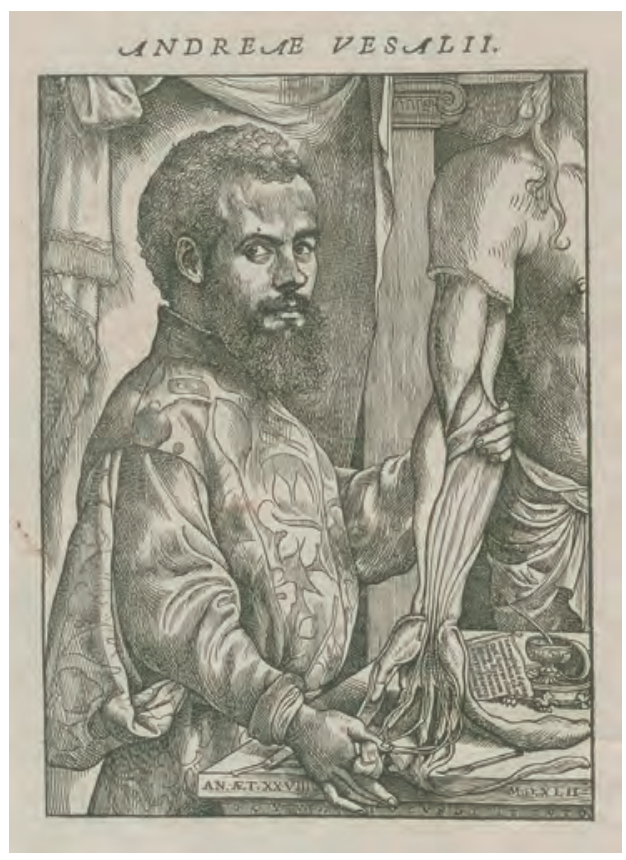

An anatomical demonstration

Engraving from Vesalius' De humani corporis fabrica

(17) Andreas Vesalius (1514-1564): De bumani corporis fabrica (1543)

Vesalius studied at Louvain and Paris, and received his doctoral degree in 1537 at Padua, where he was appointed professor the very same year. In 1544, he became court physician of Charles V, later of Philip II. He is internationally renowned for his innovative research on human anatomy.

A right-minded humanist, Vesalius adamantly advocates a reform, or perhaps rather a 'renaissance', of medicine. In his ardent plea, antiquity clearly functions as a model to be imitated: 
Quando ingeniorum fons Homerus medicum virum multis praestantiorem esse affirmat, et cum universis Graeciae poetis Podalyrium et Machaonem celebrat, hi divi Aesculapii filii non ideo praedicantur, quod febriculam, et quae sola natura absque medici auxilio levius quam illo adhibito plerunque sanat, removerent, hominumve palato in reconditis deploratisque affectibus subservirent, sed quia luxationum, fracturarum, contusionum, vulnerum et reliquarum continuitatis solutionum, sanguinisque fluxus curationi potissimum praeessent, ac spiculis, telis atque aliis eiusmodi malis (quae bella in primis inferunt, diligentemque medici operam semper expetunt) generosissimos Agamemnonis milites liberarent. (...)

Perversissima curationis instrumentorum ad varios artifices diductio, adbuc multo execrabilius naufragium ac longe atrociorem cladem praecipue naturalis philosophiae parti intulit: cui, cum hominis historiam complectatur, firmissimumque totius medicae artis fundamentum ac constitutionis initium iure habenda sit, Hippocrates et Plato tantum tribuerunt, ut illi inter medicinae partes primas esse adscribendas non dubitarint. Haec namque cum prius a medicis unice excoleretur, ipsique in hac adipiscenda omnes nervos intenderent, tum demum misere collabi coepit, cum ipsi manuum munus ad alios reiicientes, Anatomen perdiderunt.

- Préface d'André Vésale à ses livres dur l'anatomie, suivie d'une lettre à Jean Oporinus, son imprimeur. Texte introduit, établi, traduit et annoté par Louis Bakelants (Bruxelles, 1961), pp. 20-23 en 25-27.

(18) Baldasar Heseler (1508/9-1567): an anatomical demonstration by Vesalius

Baldasar Heseler was a medical student at Bologna when he made the following notes from lectures on Mondino dei Luzzi's Anatomia corporis bumani, given by Matthaeus Curtius (1475-1542), a noted Italian physician and teacher, and demonstrated by none other than Andreas Vesalius:

Instituta erat anathomè huius nostri subiecti in illo loco ubi solent rectores Medicorum eligi, satis commode et bene mensa in qua subiectum iacebat, et quattuor gradibus sedilium circumquaque ad formam 
rotundam instructo, ita ut pene ducentae personae videre anathomèn potuerunt. Nemo tamen intromittebatur, nisi primum domini anathomistae, et deinde ii qui 20 solidos persolverant. Scolaribus igitur ultra 150 praesentibus et D[omino] Curtio, Erigio, caeterisque multis aliis Curtianis Doctoribus, etc. Veniebat postremo D[ominus] Andreas Vesalius: incendebantur multae cereae candelae, ut possimus omnes videre.

Incipiebat igitur D[ominus] Vesalius: 'Scitis,' inquit, 'domini, quomodo soleant Medici et veteres et moderni corpus humanum partiri. Aegiptii enim atque Arabes incipiunt a trunco et extremitatibus, sed Galenus secundum tres ventres, quem etiam Mundinus secutus est. Sed his omissis' (Curtius enim admonebat ut ostenderet quae praelegerat) 'ad anathomiam perveniemus.' Atque tunc iam antea sectum et praeparatum erat corpus, prius rasum, lavatum et mundatum. Incipiebat igitur ab extrinseca cute, cui interius interna adhaerebat, quae vera dicitur cutis, exterior autem illa corium potius. 'Et quia,' inquit, 'non bene possunt hae duae separari, nam illa interior est nimis subtilis et vere spermatica, quae rupta aut discissa consolidari non potest.' Igitur faciebat hanc inter eas differentiam. Urebat lumine extrinsecam quae corium dicitur, et ostendebat nobis quomodo vesicaretur, cum tamen intrinseca non vesicaretur, quia magis carnosa et carniformis illa externa esset.

Postmodum pinguedo adhaerebat panniculo carnoso sub cute, qui seiunctus rasorio erat. Dixit quod pinguedo resudans ad cutem coagularetur a frigiditate cutis. Intus in ista membrana seu panniculo ostendebat nobis venarum capita nigra, et orificia nervorum tendentium pro nutricatione cutis utriusque et pro sensu interioris cutis.

Atque haec nobis ostendebat in sinistro latere. In dextro latere autem non istum panniculum seiunxerat. 'Sic,' inquit, 'solent laniatores excoriare corpora.' Demum veniebat ad anathomen musculorum quos sane artificiosissimus, summa cum industria seiunxerat, ita ut singuli substantia, magnitudo, situs, ortus et finis conspici potuit.

- Andreas Vesalius' First Public Anatomy at Bologna, 1540. An Eyewitness Report by Baldasar Heseler, Medicinae Scolaris, Together With His Notes on Matthaeus Curtius' lectures on Anatomia Mundini. Edited, with an Introduction, Translation into English and Notes by R. Eriksson, Lychnos-Bibliotek, 18 (Uppsala-Stockholm, 1959), pp. 84-87. 
(19) Gerardus Mercator (1512-1594): letter to Abraham Ortelius, 8 August 1579

Mercator was a cartographer, maker of instruments and engraver from Rupelmonde. In 1544, he was arrested on suspicion of adhering to the Protestant faith. In 1552, he left for Duisberg, where he became professor of cosmography and published his major works.

The following letter clearly reveals how intimately entangled science and politics could be in early modern times. For geo-political reasons, the Portuguese withheld their maps of certain parts of the New World. This greatly annoyed cartographers like Mercator and Ortelius:

Salve Plurimum mi D. Orteli.

Magnus cunctator sum in remittendis iis, quae mibi liberaliter concessisti, et ipse fortasse indiges, tot occupationibus cingor, ut excribere illa vix tandem datum sit. Utinam reliquam eam continentem ita descriptam haberemus. Non dubito autem quin maxima ejus pars a Portugalensibus sit descripta, postquam eam a Mexico usque in Catajum plures ac non semel peragrarunt, idque diversis itineribus; sed quae firmis praesidis et legibus sibi subjecta non habent, non lubenter opinor aliis spectanda permittunt, nam et Galli et Angli occupandis terris inhiant. Galliam universam particulatim descriptam esse, non dubito quin audieris. De Hispania simile quid tentatum intellexi, sed quousque deductum sit opus, ignoratur. Has tabulas si nancisci posses (ut posse existimo, quum in plurium evangelicorum principum manibus versentur, apud quos facilem tibi gratiam fore non dubito) maximum exoptatissimumque Theatro tuo auctarium adjungeres. Scripsit mibi filius, te aliquot Tabularum adpendicem fecisse, ejusque exemplar mibi donasse, pro quo ingentes ago gratias; spero in eo me Artesiam Hannoniamque reperturum, nam Artesiam qui excusam vidit, etiam Hannoniam mox edendam mibi dixit. Boni quaeso consule quod Tabulas Americae tamdiu retinui; si quid amplius a te habeo, aut alioqui penes me sit quo uti velis, significa, mittam continuo.

Bene vale mi Chariss. D. Orteli. Duysburgi 8 Aug.) 579. 
Tuus perpetuo

Gerardus Mercator

Amico singulari D. Abrahamo Ortelio

- M. Van Durme (ed.), Correspondance Mercatorienne (Anvers, 1959), pp. 155-156, no 140.

(20) Ferdinand Verbiest (1623-1688): Astronomia Europaea (1687)

The Jesuit Verbiest was a missionary and an astronomer. In 1659, he travelled to China where he constructed, with the approval of the Chinese emperor, various new instruments that enabled more exact measuring.

In the 17th century, the Southern Netherlands were at the head of mathematical and astronomical research. Verbiest made an important contribution to it, not so much as an original thinker, but rather as an inventive engineer. Probably as early as 1665, he built the first experimental models for an automobile driven by steam a truly revolutionary achievement. At the beginning of his 'European Astronomy', Verbiest explains how he managed to gain the trust of the Chinese emperor. On 26 December 1668, he was summoned to the emperor, together with his European colleagues and his Chinese opponents:

Imperator, qui iam satis multam de me notitiam habebat, statim me primo loco et ante omnes in conspectu suo statuendum iussit, ac benigno vultu interrogans, estne, inquit, signum aliquod manifestum, quo oculis nostris demonstrari possit, Calendarii calculum convenire vel non convenire cum caelo? Ego statim respondi, hoc facile demonstrari posse et quidem ab omni saeculo; eam tantum ob causam instrumenta astronomica exposita esse, uti adhuc hodie in specula astroptica Pekinensi videre est, ut scilicet illi, quibus ob graviora imperii negotia astronomiae vacare non licet, aperte videant, utrum calculus a caelo differat, necne. Et cum sol inter omnes planetas sit maxime conspicuus, sic et nunc 
coram oculis Maiestatis Tuae, erecto quocunque stylo, sive etiam mensa aut sedili quopiam in medio huius atrii posito, ego paratus sum calculo praedicere quam longam umbram, stylus quicunque datus, ad boram a Maiestate Tua assignandam, projecturus sit. Ex data autem longitudine umbrae manifeste arguitur altitudo solis in caelo ad eandem horam quae quotidie diversa est. Ex altitudine vero solis tali die et hora determinata aperte cognoscitur locus solis in zodiaco, adeoque an calendarii calculus invento iam loco caelesti respondeat.

Hoc meum responsum tam amoenum et optatum visum est Imperatori, quam triste et fatale adversariis nostris. Quos cum Imperator interrogaret, num et ipsi hanc umbrae calculandae artem scirent, Mabumetanus ille, non minus audacter quam temere, respondit se eam scire, illamque certam esse regulam, qua verum a falso manifeste discerni possit. Mox Imperatori iterum interroganti, num et ipsi sustentare possent astronomiam Europaeam, impudentissimus ille impostor Yangquangsenius ita statim reposuit: nullo modo, inquit, convenit Imperii Sinico-Tartarici felicitati, ut Maiestas Tua vel scientiis vel hominibus Europaeis utatur, et cum blasphemo ore multa contra religionem nostram inciperet evomere.

Imperator repente mutato in severitatem vultu silentium illi imponens dixit: 'Hoc ipso die mandatum a me fuit, ut priori omni inimicitia deposita, communi consilio de optima astronomiae lege introducenda ageretis, et tu etiam coram me nunc in haec verba erumpis?' Et post pauca haec subiunxit: 'Tu porrecto saepius libello supplice petiisti, ut homines astronomiae peritos toto Imperio quaerendos iuberem, et ecce intra hos quatuor annos quaesiti et nulli reperti sunt. Nunc autem, cum Ferdinandus Verbiest (me Sinico meo nomine appellabat) astronomiae peritissimus in hac aula Pekinensi moraretur, tu mibi nomen illius occultasti. Aperte patet te esse bominem valde abjectum, nullius fidei, habentem animum dolo et invidia plenum.'

- Noël Golvers - Ulrich Libbrecht, Astronoom van de Keizer. Ferdinand Verbiest en zijn Europese Sterrenkunde (Leuven, 1988), pp. 126-127; Noel Golvers, The 'Astronomia Europaea' of Ferdinand Verbiest, S.J. (Dillingen, 1687). Text, Translation, Notes and Commentaries, Monumenta Serica - Monograph Series, 28 (Nettetal, 1993), pp. 352-354. 


\subsection{The art of printing}

(21) Augerius Busbequius: Legationis Turcicae epistolae quatuor (1589)

Busbequius's letters clearly reveal how much respect the Turks showed for European culture; in their great admiration they were eager to take over all kinds of European inventions - with the notable exception of the printing press and the clock:

Non facile gentem aliam minus piguit aliorum bene inventa ad se transferre. Testes maiores minoresque bombardae multaque alia, quae a nostris excogitata, ipsi ad se avertunt. Ut libros tamen typis excuderent, horologia in publico baberent, nondum adduci potuerunt, quod scripturam, hoc est suas literas sacras, non amplius scripturam fore, si excuderetur, et si horologia publice haberentur, aliquid de aedituorum suorum et prisci ritus auctoritate deminutum iri arbitrarentur; alias plurimum tribuere soliti vetustis aliorum institutis paene vel cum aliqua religionis suae iniuria, de vulgo dico.

- Augerius Gislenus Busbequius, Legationis Turcicae epistolae quatuor - Vier brieven over het gezantschap naar Turkije. Uitgave, inleiding en aantekeningen van Zweder von Martels, vertaling van Michel Goldsteen (Hilversum, 1994), ep. 3, pp. 218-219.

\subsection{The visual arts}

(22) Peter Paul Rubens (1577-1640): letter to Franciscus Junius, 1 August 1637

Rubens has read with great interest the recently published treatise De pictura veterum of Franciscus Junius Junior (1589-1677), a philologist from the Northern Netherlands. As a humanist, he is impressed by the author's erudition. As a painter, however, he is not entirely satisfied. Rubens takes the liberty of explaining why: 
Liber iste D[ominationis] V[estrae] vere promus condus est uberrimus omnium exemplorum, sententiarum et dogmatum quae a veteribus uspiam sparsim ad dignitatem et lucem Artis Pictoriae pertinentia, litteris consecrata maximo nostro emolumento bactenus perennarunt. (...) Sed quoniam exempla illa veterum Pictorum phantasia tantum et pro cuiusque captu magis aut minus assequi possumus, vellem equidem eadem diligentia similem quandoque tractatum excudi posse de Picturis Italorum, quorum exemplaria sive prototypa adbuc hodie publice pros tant, et digito possunt monstrari et dicier 'haec sunt'. Nam illa quae sub sensum cadunt, acrius imprimuntur et haerent, et exactius examen requirunt, atque materiam uberiorem proficiendi studiosis praebent, quam illa quae sola imaginatione tanquam somnia se nobis offerunt, et verbis tantum adumbrata ter frustra comprensa (ut Orpheum Euridices Imago) eludunt saepe, et sua quemque spe frustrantur. Quod experti dicimus, nam quotusquisque nostrum si praeclarum aliquod Apellis aut Timanthis opus a Plinio aut aliis auctoribus graphice descriptum, pro rei dignitate oculis subicere tentaturus, aliquid non insulsum aut a veterum maiestate non alienum praestabit, sed genio suo quisque indulgens, musteum aliquid pro Opimiano illo dulceamaro promit, et iniuriam magnis illis manibus affert, quos ego veneratione summa prosequor, et vestigia euntium potius adoro, quam vel sola cogitatione assequi me posse ingenue fateor.

Ick bidde mijn Heere believe mij wel af te nemen 't gene ick met vriendelijcke liberteit ben seggende, met hope dat UE. ons naar soo goeden promulsidem niet en sal weigeren ipsum caput coenae, daer wij allegaeder seer naer verlangen: want tot noch toe en heeft ons niemant onsen appetijt voldaen, van alle die gene die alsulcken materie getracteert hebben, nam oportet venire ad individua, ut dixi. (I pray, my Lord, that you may take well what I have said with friendly liberty. You have served us such a delicious entrée, so I bope you will not refuse us le plat de résistance, for which we long so much. For thus far, of all those authors who have discussed this matter, no one has satisfied our appetite. For, as I have said, it is necessary to come to individual cases). 
- Franciscus Junius, De pictura veterum libri tres (Roterodami 1694). Edition, traduction et commentaire du livre I par Colette Nativel, Travaux du Grand Siècle, 3 (Genève, 1996), pp. 109-110; The Letters of Peter Paul Rubens. Translated and Edited by Ruth Saunders Magurn (Evanston, Ill., 1991 (= 1955), pp. 406-408.

\subsection{Music}

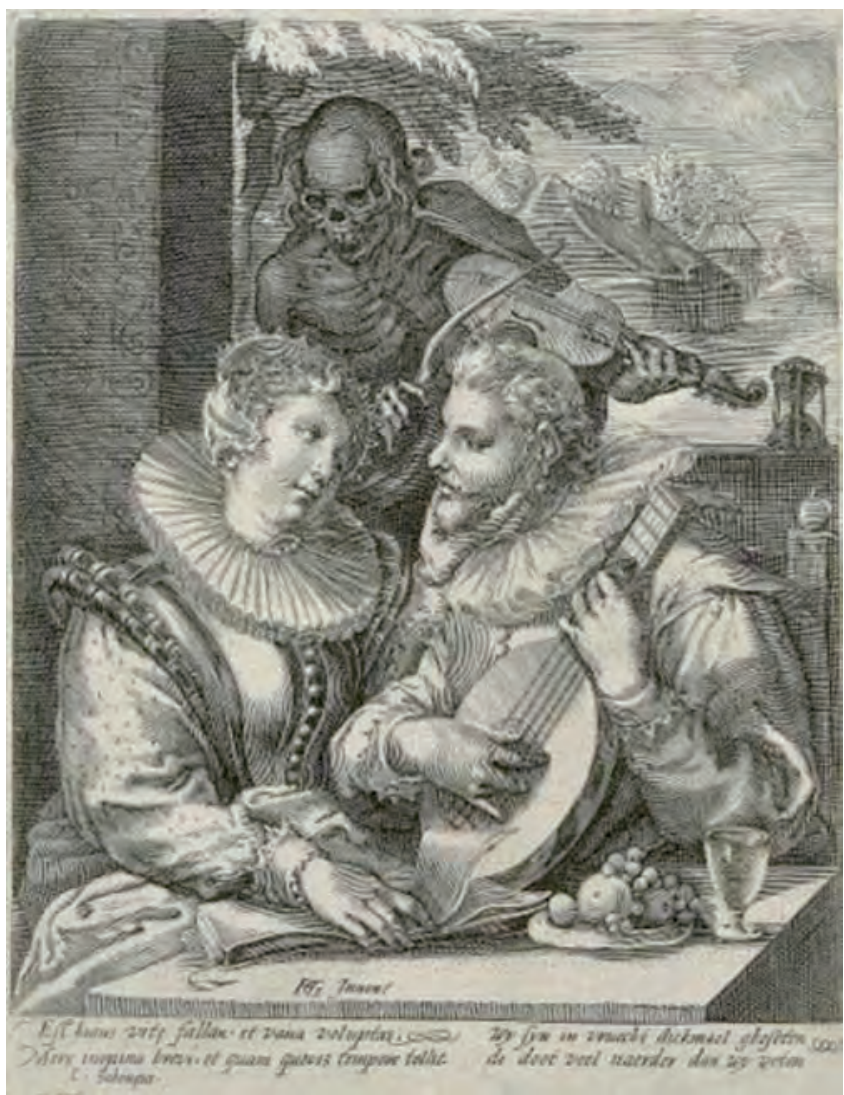

Constantijn Huygens enchanting his beloved one with his music Engraving from Huygens' Zedeprinten (1623)

http://www.fransmensonides.nl/5laatsteprinten/verrastdoordood.htm

\section{(23) Constantijn Huygens: playing music together}

As mentioned above, Huygens was not only a diplomat, but also a composer and a talented amateur musician. He often played mu- 
sic together with his friends. For the 'exceptionally learned woman' Anna Maria van Schurman (1607-1678) from Utrecht, he composed the following invitational poem, asking her to receive him at home in order to play music together. Huygens hopes that her brother Johan Godschalk van Schurmen (1605-1664) and Gisbertus Voetius (1589-1676), a professor of theology, will join them as well, and the same goes for the singer Utricia Ogle, who was married with William Swan, an English captain serving Holland, and who also lived in Utrecht:

\section{AD EANDEM REDUX}

Fugi nuper: an bic fuerit pudor, an vis maior,

Ambigo: si bene rem pondero, utrumque fuit.

Nunc procul et pudor est, procul et vis maior et Aulae

Assiduum, compes aurea, seruitium.

Libera per vacuum pono vestigia; quo me

Fata ferunt, quo me ventus et aura, feror.

Et feror, en, Fatis, aurâ ventoque secundis

Quo si saepe ferar saepius ire velim.

In Musas, mea Musa, tuas: ignosce molesto:

(10) Aut leuis, ut monui, est, aut breuis iste dolor.

Quod si tam superos habeas in vota benignos,

Ut mea se nobis tertia jungat $O$ lor,

Quartus erit Cycnus, quinto fortassis amabunt

Voetius et sexto Frater adesse loco.

(15) Septima ne jubeas accedat lingua, nec auris

Septima; sat seni musica turba sumus,

Et faciam, si quid possunt mea Carmina, dicas,

Hei mihi, quam leuis est, quam breuis iste dolor!

Inter Rhenam et Arnhemum 10. Octob.

Constantijn Huygens, Gedichten. Ed. J.A. Worp (Groningen, 1893-1899), Vol. 4, pp. 240-241; J. Bloemendal, Een handvol Huygens. Vijf Latijnse gedichten van Constantijn Huygens vertaald en toegelicht (Voorthuizen, 1997), pp. 12-13, 33-34. 


\subsection{Religion}

The Northern Netherlands have sometimes been characterized as a country filled with theologians. True enough, nearly everyone had an opinion about a religious problem. Two famous theologians from the North deserve to be mentioned here: Erasmus and Gisbertus Voetius (1589-1676).

\section{(24) Desiderius Erasmus: Enchiridion (1503)}

Erasmus was first and foremost a theologian. One of his best-known works as a theologian is the Enchiridion militis christiani (Manual/Hand weapon of the Christian soldier). It first appeared in 1503 as part of the Lucubrationes (Works written at lamplight). However, it remained largely ignored, until it was published as a separate treatise, with a dedicatory letter addressed to Paul Volz, in 1518. From that time onwards, the treatise became widely known, and for a good reason: it can be rightly considered as the manifesto of Northern biblical humanism. In it the works of classical Antiquity are regarded as a propaedeutic, an introduction and preparation to the study of the Bible and the Church Fathers - a combined study whose primary aim is to foster inner piety:

Ergo si te totum studio scripturarum dedicabis, si in lege domini meditaberis die ac nocte, non timebis a timore nocturno sive diurno, sed ad omnem hostium insultum munitus atque exercitatus eris. Neque equidem usquequaque improbaverim ad hanc militiam velut tirocinio quodam praeludere in litteris poetarum et philosophorum gentilium, modo modice ac per aetatem quis eas attingat et quasi in transcursu arripiat, non autem immoretur et veluti ad scopulos Sireneos consenescat. Nam ad haec quoque studia vocat divus Basilius adolescentulos, quos ad Christianos mores instituit, et ad Musas revocat Licentium suum noster Augustinus. (...) Verum nolim te cum gentilium litteris gentilium et mores haurires. Alioqui permulta reperies et illic ad recte vivendum conducibilia neque aspernandum, quod etiam ethnicus 
auctor bene monet, quandoquidem nec Moses Iethro soceri consilium est aspernatus. Fingunt illae litterae vegetantque puerile ingenium atque ad divinarum scripturarum cognitionem mire praeparant, ad quas ilico pedibus manibusque illotis irrumpere patrie sacrilegii genus est. Hieronymus eorum impudentiam taxat, qui modo a saecularibus studis profecti audent divinas tractare scripturas, at quanto faciunt impudentius, qui ne gustatis quidem illis istuc ipsum audent. Sed uti divina scriptura non multum habet fructus, si in littera persistas haereasque, ita non parum utilis est Homerica Vergilianaque poesis, si memineris eam totam esse allegoricam. Id quod nemo negabit, qui modo veterum eruditionem vel summis labiis degustarit. Obscoenos autem poetas suaserim omnino non attingere aut certe non introspicere penitius, nisi forte noveris descripta magis horrere vitia et contentione turpium vehementius amare honesta.

E philosophis autem Platonicos te sequi malim, propterea quod cum plerisque sententiis tum ipso dicendi charactere quam proxime ad propheticam evangelicamque figuram accedunt. Breviter omnem ethnicam litteraturam delibare profuerit, si quidem id fiat, ut dixi, et annis idoneis et modice, tum cautim et cum delectu, deinde cursim et peregrinantis, non habitantis more, postremo, quod est praecipuum, si omnia ad Christum referantur. Sic enim mundis omnia munda sunt, cum immundis contra nibil sit mundum.

- Erasmus, Ausgewählte Werke, eds. Annemarie and Hajo Holborn, (München, 1964 [= 1933]), pp. 31-32; John W. O'Malley (ed.), Collected Works of Erasmus 66 (Toronto, Buffalo, London, 1988), pp. 33-34.

\subsection{Philosophy}

Philosophy and philosophers found rather fertile soil in the Netherlands. It was there that Justus Lipsius promoted a practical kind of Stoic philosophy; it was there that the French philosopher René Descartes spent several years of his adult life; and it was there that the radical philosopher Baruch de Spinoza lived and worked. 
In 1677, Baruch (also called Benedictus) de Spinoza published his Ethica Ordine Geometrico Demonstrata ('Ethics demonstrated by means of geometric method'). In this work, Spinoza tackled, among other things, the problem of free will:

Propositio XXXII. Voluntas non potest vocari causa libera; sed tantum necessaria.

Demonstratio. Voluntas certus tantum cogitandi modus est, sicuti intellectus; adeoque (per Prop. 28) unaquaeque volitio non potest existere, neque ad operandum determinari, nisi ab alia causa determintur, et haec rursus ab alia, et sic porro in infinitum. Quod si voluntas infinita supponatur, debet etiam ad existendum, et operandum determinari a Deo, non quatenus substantia absolute infinita est; sed quatenus attributum habet, quod infinitam, et aeternam cogitationis essentiam exprimit. (per Prop. 23.) Quocumque igitur modo, sive finita, sive infinita concipiatur, causam requirit, a qua ad existendum, et operandum determinetur; adeoque (per Defin. 7.) non potest dici causa libera; sed tantum necessaria, vel coacta. Q.E.D.

Corollarium I. Hinc siquitur $I^{o}$. Deum non operari ex libertate voluntatis.

Corollarium III. Sequitur IIO. voluntatem, et intellectum ad Dei naturam ita sese habere, ut motus, et quies; et absolute, ut omnia naturalia, qua (per Prop. 29.) a Deo ad esitendum, et operandum certo modo determinari debent. Nam volutnas, ut reliqua omnia, causa indiget, a qua ad existendum, et operandum certo modo determinetur. (...)

- $\quad$ Spinoza, Opera - Werke. Vol. 2: Tactatus de Intellectus Emendatione - Ethica, ed. Konrad Blumenstock (Darmstadt, 1989 [= 1967]). 


\section{Society and politics}

\subsection{Towards a social Europe?}

(26) Joannes Ludovicus Vives: De subventione pauperum sive de bumanis necessitatibus (1526)

In this pioneering treatise, Vives stresses that it is a fundamental task of the government to combat poverty. In doing so, the government is allowed to take harsh measures. Forced employment of beggars, expulsion of unemployed foreigners: according to Vives, these are perfectly acceptable policy tools:

Ex mendicis validis alienigenae remittendi ad suas civitates (quod etiam iure Caesareo cavetur), adiecto viatico; nam inhumanum esset egenum sine viatico in iter mittere et qui hoc faceret, quid aliud quam iuberet rapere? Inter propinquos et affines tum pudebit eos magis segnitiae et turpitudinis vitae, tum facilius subsidium invenient quam foris. Si vero ex vicis aut oppidulis sine bello afflictis, tum reputandum quod Paulus docet, inter baptizatos in sanguine Christi iam nec Graecum esse nec barbarum nec Gallum nec Flandrum, sed novam creaturam, pro indigenis habendi.

Indigenae rogandi an artem norint ullam. Qui nullam norunt, si aetate sunt idonea, edocendi ad quam dicent se maxime propensos, modo liceat; sin secus, ad similem quampiam; ut cui non licebit suere vestes, suat quas caligas nominant; sin est provectior aut ingenio nimis tardo, facilior aliqua tradatur, denique ea quam nemo non paucis diebus perdiscat: fodere, haurire aquam, baiulare, monotrochon trabere, apparere magistratui, viatorem esse, aliquo proficisci cum litteris aut mandatis, equos agere perpetuarios.

- Juan Luis Vives, De subventione pauperum sive de humanis necessitatibus libri II. Introduction, Critical Edition, Translation and Notes by C. Matheeussen and C. Fantazzi, with the assistance of J. De Landtsheer, Selected Works of J.L. Vives (Leiden-Boston: Brill, 2002), 2,3,3-4, pp. 100-101. 


\subsection{Emancipation of women?}

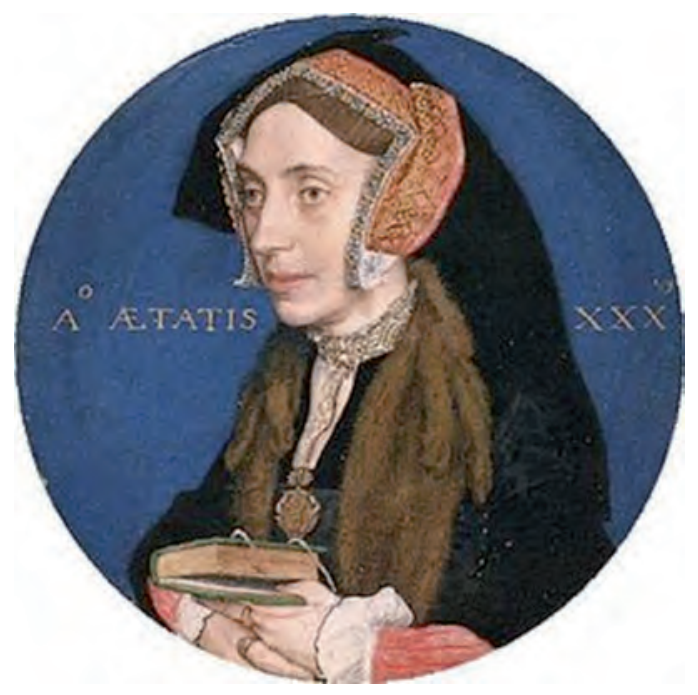

A real-life model for Erasmus' Magdalia: Thomas More's learned daughter Margaret Roper Painting by Hans Holbein the Younger http://en.wikipedia.org/wiki/File:Margaret_ Roper,_by_Hans_Holbein_the_Younger.jpg

(27) Desiderius Erasmus: Colloquium abbatis et eruditae (1524)

In March 1524, Erasmus published a pithy dialogue in which he criticized the decadence of some abbots and advocated proper intellectual education of women - a rare, but by no means unique plea in the 16th century. In the dialogue, Magdalia, a learned woman with a sharp intellect and an even sharper tongue, crosses swords with Antronius, a stupid, conceited abbot who attaches greater importance to his pleasures than to wisdom and piety. As is to be expected, the conversation between them is doomed to fail: Magdalia is speaking to deaf ears:

A. Nescio quomodo fit, ut quemadmodum clitellae non conveniunt bovi, ita nec literae mulieri.

M. Atqui negare non potes, quin magis quadrent clitellae bovi, quam mitra asino aut sui. Quid sentis de Virgine matre? 
A. Optime.

M. Nonne versabatur in libris?

A. Versabatur, at non in istis.

M. Quid igitur legebat?

A. Horas canonicas.

M. Ad quem usum?

A. Ordinis Benedictini.

M. Sit ita sane. Quid Paula et Eustochium, nonne versabantur in sacris libris?

A. Verum istuc nunc rarum est.

M. Sic olim rara avis erat abbas indoctus, nunc nibil vulgatius. Olim principes et Caesares eruditione non minus quam imperio praeminebant. Neque tamen usque adeo rarum est, quam tu putas: sunt in Hispania, sunt in Italia non paucae mulieres apprime nobiles quae cum quovis viro queant contendere; sunt in Anglia Moricae, sunt in Germania Bilibaldicae et Blaurericae. Quod nisi caveritis vos, res eo tandem evadet, ut nos praesideamus in scholis theologicis, ut concionemur in templis. Occupabimus mitras vestras.

A. Ista Deus avertat.

M. Imo vestrum erit hoc avertere. Quod si pergetis, ut coepistis, citius anseres concionaturi sint, quam vos mutos pastores ferant. Videtis iam inverti mundi scaenam. Aut deponenda est persona, aut agendae sunt suae cuique partes.

A. Unde incidi in hanc feminam? Si quando vides nos, ego te suavius accipiam.

M. Quibus modis?

A. Saltabimus, bibemus affatim, venabimur, ludemus, ridebimus.

M. Mibi quidem iam nunc ridere libet.

- C. Matheeussen, J. De Landtsheer, T. Van Houdt and L. Verachten (eds.), Erasmus en Vives over vrouwen en opvoeding. Een didactisch dossier voor de derde graad, Aulos. Studies over oude talen en antieke cultuur (Leuven, 2001), pp. 78-79; Desiderius Erasmus, Gesprekken - Colloquia. Vertaald en toegelicht door Jeanine De Landtsheer (Amsterdam, 2001), pp. 231-232; Craig R. Thompson (ed.), Collected Works of Erasmus, vol. 39. Colloquies (London, 1997), pp. 504-505. 
From 1632 onwards, Anna Maria van Schurman was engaged in a hot debate with the theologian André Rivet about the question of whether or not women were suitable for study and science. Nine years later, she wrote an extensive treatise on the matter that made her world-famous: the Dissertatio de Ingenii Muliebris ad Doctrinam \& meliores Litteras aptitudine. Adopting a rigorous, strictly 'scientific' line of reasoning, Van Schurman consciously appropriates a typically male language and genre in order to meet her male discussion partners. In so doing, she convincingly proves that there is at least one woman who deserves to be admitted to the closed circle of academic, male conversation and discussion.

Sit igitur Thesis nostra. Foeminae Christianae convenit studium Litterarum.

Cui confirmandae argumenta haec adferimus primo ex parte Subiecti:deinde etiam, Praedicati.

I. Argumentum ex proprio Subiecti.

Cuicunque natura indita sunt principia, seu potentiae principiorum omnium artium, ac scientiarum, ei conveniunt omnes artes ac scientiae: Atqui foeminis natura indita sunt principia seu potentiae principiorum omnium artium ac scientiarum. Ergo Foeminis conveniunt omnes artes ac scientiae.

Propositio probatur: quia cui conveniunt principia seu potentiae principiorum, ei convenit notitia conclusionum, quae sua natura ex iisdem educuntur.

Assumptio probari potest, tum ex proprio formae huius Subiecti, sive rationis humanae: tum ex ipsis actibus seu effectis, siquidem foeminas actu scientias et artes quaslibet addiscere manifestum est: actus vero nulli sine principiis esse possunt.

II. Argumentum a proprio Subiecti.

Cui natura inest scientiarum artiumque desiderium, ei conveniunt scientiae et artes: Atqui Foeminae natura inest scientiarum artiumque desiderium. Ergo.

Maioris ratio patet, quia Natura nibil facit frustra. 
Minor probatur, quia quod inest toti speciei, inest etiam singulis individuis. Atqui omnis homo (ut exserte statuit Philosophus Metaphysic. lib. 1. cap.2) natura scire desiderat.

- Anna Maria van Schurman, Opuscula Hebraea, Graeca, Latina, Gallica, Prosaica et Metrica (Leiden: Elzevier, 1650), pp. 33-35; Anna Maria van Schuurman, Verhandeling over de aanleg van vrouwen voor wetenschap (Groningen, 1996); Anna Maria van Schurman, Whether a Christian Woman Should Be Educated and Other Writings from Her Intellectual Circle. Edited and Translated by Joyce L. Irwin, The Other Voice in Early Modern Europe (Chicago-London, 1998), pp. 27-28.

\subsection{Good governance}

(29) Justus Lipsius: Politicorum sive civilis doctrinae libri sex (1589)

For decades, Lipsius's treatise was one of the most influential political manuals in Europe. It was eagerly read, extensively commented upon, and diligently used as a political compass in both the Protestant and Catholic world. The core of the treatise is to be found in the fourth book, which focuses on the many serious problems a ruler is inevitably confronted with. One of those problems concerns the collection of taxes. Following Erasmus's lead, Lipsius stresses the need for good governance - taxes should be spent with frugality and only after due consideration:

At quartum fomentum, Dispensatio. Eam restrictam utilemque esse suadeo, et qualis in familia boni patris. Exiguam in publicum impensam quis gravabitur, si videant te continenter sobrieque viventem, ac nibil vane effundentem? Scient enim vere in publicum tribui: teque esse dumtaxat custodem et dispensatorem, ut communium bonorum, non ut tuorum. Ita debes. Non privatim largiri, quod avidius de republica sumas, et explere hiantes in magna fortuna amicorum cupiditates. Non per delicias aut sumptus, pecuniae illudere: ut quas honeste habere et uti licet, per turpitudinem abuti properes. Absit. Alexander Romanus tibi praeerat: qui aurum et argentum raro cuiquam nisi militi divisit, nefas esse dicens, ut dispensator publicus, in delectationes suas suorumve converteret, id quod 
Provinciales dedissent. O virum, o Principem! Non illos tyranniones, qui divitiarum et pecuniae non alium fructum putant, quam profusionem.

- Justus Lipsius, Politica. Six Books of Politics or Political Instruction, edited, with translation and introduction, by Jan Waszink, Bibliotheca Latinitatis Novae (Assen, Royal Van Gorcum, 2004), pp. 476-479.

\subsection{War...}

(30) Hugo Grotius: De iure belli ac pacis (1625)

Grotius is rightly hailed as the founder of modern international law. In his seminal treatise on the rights of war and peace, he defines the minimal rules that should govern the relations between states and peoples. Grotius was by no means a pacifist. According to him, waging a war is a natural right, albeit a right constrained by numerous conditions:

Sileant ergo leges inter arma, sed civiles illae et iudiciariae et pacis propriae, non aliae perpetuae et omnibus temporibus accommodatae. Optime enim dictum est a Dione Prusaeensi, inter hostes scripta quidem iura, id est, civilia non valere, at valere non scripta, id est, ea quae natura dictat, aut gentium consensus constituit. Docet boc vetus illa Romanorum formula: eas res puro pioque duello quaerendas censeo. Iidem veteres Romani, ut Varro notabat, bella tarde et nulla licentia suscipiebant, quod bellum nullum nisi pium putabant geri oportere. Camillus iuste non minus, quam fortiter bella gerenda dicebat: Africanus populum Romanum et suscipere iuste bella, et finire. Apud alium legas: Sunt et belli, sicut et Pacis iura. Alius Fabricium miratur ingentem virum, et quod difficillimum est in bello innocentem, et qui aliquid esse crederet et in hostem nefas.

- Hugo Grotius, De iure belli ac pacis libris tres, ed. P.C. Molhuysen (Leiden, 1919), p. 11; Hugo de Groot, Denken over oorlog en vrede. Uitgegeven, ingeleid en van aantekeningen voorzien door A.C. Eyffinger en B.P. Vermeulen, Geschiedenis van de wijsbegeerte in Nederland (Baarn, 1991), pp. 49-50; Hugo Grotius, The Rights of War and Peace, book I. Edited and with an Introduction by Richard Tuck. From the Edition by Jean Barbeyrac, Natural Law and Enlightenment Classics (Indianapolis, 2005), pp. 102-103 (1738 translation by John Morrice). 
(31) Leonardus Lessius (1554-1623): Commentarius in secundam secundae Divi Thomae (1592)

The Jesuit Lessius was an important moral theologian from Brecht near Antwerp. He was renowned for his sharp observations about, and penetrating insights into, business ethics and international law.

In his lectures on Thomas Aquinas's Summa theologiae, Lessius paid much attention to the question of just war (bellum iustum). Following the lead of the so-called School of Salamanca, he developed a balanced doctrine that would influence natural-law thinkers such as Hugo Grotius. In the following passage, Lessius discusses whether or not a ruler is allowed to launch a war in order to protect the innocent subjects of another state:

Petes utrum non sint alii tituli belli inferendi praeter iniuriam? Respondeo: quidam dicunt peculiariter fideles habere quosdam alios titulos, quorum quintus est innocentium defensio, ut si alicubi sacrificentur innocentes vel mactentur, possunt bello peti. Sed boc bellum non tam est aggressivum de quo disputamus, quam defensivum. Non enim princeps Christianus potest vindicare iniurias innocentibus in alia Republica nisi id ipsi velint (...). Potest tamen probibere ne fiant, et cogere ut abstineant ab iniuria, quod est defendere innocentes. Quivis enim potest defendere innocentem, praesertim in is in quibus ipse non potest cedere iure suo, nempe in vita et integritate membrorum, quamvis id ipse innocens non requirat. In ceteris autem nisi ipse innocens censeatur petere, ut si alicui auferantur facultates, non licet eum defendere si ipse nolit, quia si non vult defendi, sponte vult eas amittere a quo non potest probiberi. Nam potest cedere iure suo, quod habet ad eas retinendas.

Praeter hos dictos titulos non occurrunt alii ullo modo probabiles. Ex quibus patet solam iniuriam illatam esse iustum titulum ad bellum inferendum ac proinde omnem titulum belli indicendi qui convenit Christianis posse etiam convenire ethnicis suo modo.

- J. Bittremieux, Lessius et le droit de guerre. Contribution à l'histoire des doctrines théologiques sur la guerre (Bruxelles, 1920), pp. 115-117. 


\section{5.... and peace}

\section{(32) Desiderius Erasmus: Querela pacis (1516)}

The dream of a unified and peaceful Europe... According to Erasmus, narrow-minded nationalism and human solidarity are incompatible. That is the reason why he makes a passionate plea in favour of collaboration and concord among all Europeans. The unified Europe which Erasmus has in mind is thoroughly and exclusively Christian; a multicultural or multi-religious society was inconceivable to him:

Magna pars pacis est, ex animo velle pacem. Quibus enim pax vere cordi est, hi omnes pacis occasiones arripiunt: quae obstant, aut negligunt, aut amoliuntur, permulta ferunt, dum tantum bonum sit incolume. Nunc ipsi bellorum seminaria quaerunt: quod ad concordiam facit, elevant, aut dissimulant etiam; quod ad bellum tendit, ultro exaggerant, exulcerantque. Pudet referre, ex cuiusmodi nugis, quantas excitent tragoedias, et ex quam minuta scintillula, quae rerum tempestates exoriantur. Tunc illud iniuriarum agmen venit in mentem, et suum quisque malum sibi exaggerat. At benefactorum interim profunda oblivio, ut iures adfectari bellum. Et saepe Principum privatum quiddam est, quod orbem ad arma compellit. At plus quam publicum esse debet, ob quod bellum suscipiatur. Quin ubi nibil subest causae, ipsi dissidiorum causas sibi fingunt, regionum vocabulis ad odiorum alimoniam abutentes: et hunc stultae plebis errorem alunt Magnates, et in suum abutuntur compendium, alunt Sacerdotes quidam. Anglus hostis est Gallo, nec ob aliud, nisi quod Gallus est. Scoto Britannus infensus est, nec aliam ob rem, nisi quod Scotus est. Germanus cum Franco dissidet, Hispanus cum utroque. O pravitatem, disiungit inane loci vocabulum, cur non potius tot res conciliant? Male vis Britannus Gallo. Cur non potius bene vis homo homini? Christianus Christiano? Cur res frivola plus apud istos potest, quam tot naturae nexus? tot Christi vincula? Locus corpora dirimit, non animos.

- Erasmus von Rotterdam, Ausgewählte Schriften, 5. Herausgegeben von Werner Welzig. Übersetzt, eingeleitet und mit Anmerkungen versehen von Gertraud Christian (Darmstadt, 1990), pp. 428-431; A.H.T. Levi, Collected Works of Erasmus, 27 (Toronto -Buffalo - London, 1986), pp. 314-315. 